\title{
Full-Rank Perfect Codes over Finite Fields
}

\author{
Alexander M. Romanov
}

\begin{abstract}
In this paper, we propose a construction of fullrank $q$-ary 1-perfect codes over finite fields. This construction is a generalization of the Etzion and Vardy construction of fullrank binary 1-perfect codes (1994). Properties of $i$-components of $q$-ary Hamming codes are investigated and the construction of full-rank $q$-ary 1-perfect codes is based on these properties. The switching construction of 1-perfect codes are generalized for the $q$-ary case. We give a generalization of the concept of $i$-component of 1-perfect codes and introduce the concept of $(i, \sigma)$-components of $q$-ary 1-perfect codes. We also present a generalization of the Lindström and Schönheim construction of $q$-ary 1-perfect codes and provide a lower bound on the number of pairwise distinct $\boldsymbol{q}$-ary 1-perfect codes of length $\boldsymbol{n}$.
\end{abstract}

Index Terms-Full-rank perfect codes, Hamming codes, $i$ components, $(i, \sigma)$-components, $q$-ary perfect codes, switching construction.

\section{INTRODUCTION}

$\mathbf{L}$ ET $\mathbb{F}_{q}^{n}$ be a vector space of dimension $n$ over the finite field $\mathbb{F}_{q}$ of order $q$. An arbitrary subset $\mathcal{C}$ of $\mathbb{F}_{q}^{n}$ is called a $q$-ary code of length $n$. The vectors in $\mathcal{C}$ are called codewords. A code $\mathcal{C}$ is called linear if it is a linear space over $\mathbb{F}_{q}$. The orthogonal complement $\mathcal{C}^{\perp}$ is the dual code of $\mathcal{C}$. A generator matrix of a linear code $\mathcal{C}$ is matrix whose rows form a basis for $\mathcal{C}$. A parity-check matrix of a linear code $\mathcal{C}$ is matrix whose rows form a basis for $\mathcal{C}^{\perp}$. The (Hamming) distance between two vectors $\mathbf{x}, \mathbf{y} \in \mathbb{F}_{q}^{n}$ is the number of coordinates in which they differ, and it is denoted by $d(\mathbf{x}, \mathbf{y})$. The (Hamming) weight $w t(\mathbf{x})$ of a vector $\mathbf{x} \in \mathbb{F}_{q}^{n}$ is the number of nonzero coordinates in $\mathbf{x}$. The (minimum) distance $d(\mathcal{C})$ of a code $\mathcal{C}$ is the smallest distance between distinct codewords. The linear code $\mathcal{C} \subseteq \mathbb{F}_{q}^{n}$ has parameters $[n, k, d]_{q}$ if $\mathcal{C}$ has length $n$, dimension $k$, and minimum distance $d$. The nonlinear code $\mathcal{C} \subseteq \mathbb{F}_{q}^{n}$ has parameters $(n,|\mathcal{C}|, d)_{q}$ if $\mathcal{C}$ has length $n$, number of codewords $|\mathcal{C}|$, and minimum distance $d$.

We define a map $p: \mathbb{F}_{q}^{n} \rightarrow \mathbb{F}_{q}$. If $\mathbf{x}=\left(x_{1}, x_{2}, \ldots, x_{n}\right) \in$ $\mathbb{F}_{q}^{n}$ then $p(\mathbf{x})=\sum_{i=1}^{n} x_{i}$. If $\mathcal{C}$ is an $(n,|\mathcal{C}|, d)_{q}$ code over $F_{q}$ then the extended code $\widehat{\mathcal{C}}$ to be the code

$$
\widehat{\mathcal{C}}=\{(\mathbf{x} \mid p(\mathbf{x})): \mathbf{x} \in \mathcal{C}\} .
$$

The extended code $\widehat{\mathcal{C}}$ is an $(n+1,|\mathcal{C}|, \hat{d})_{q}$ code, where $\hat{d}$ equals $d$ or $d+1$.

If $\mathcal{C}$ is a $q$-ary $(n,|\mathcal{C}|, d=2 t+1)_{q}$ code, then we have

$$
|\mathcal{C}| \sum_{k=0}^{t}\left(\begin{array}{l}
n \\
k
\end{array}\right)(q-1)^{k} \leq q^{n} .
$$

The work was supported by Russian Foundation for Basic Research under grant 11-01-00997.

The author is with the Sobolev Institute of Mathematics, Siberian Branch of the Russian Academy of Sciences, 4 Academician Koptyug avenue, 630090 Novosibirsk, Russia (e-mail: rom@math.nsc.ru).
If the equality occurs, then $\mathcal{C}$ is called a perfect code. The inequality (1) is known as the Sphere-Packing Bound.

A $q$-ary perfect code $\mathcal{C}$ with parameters $(n,|\mathcal{C}|, 3)_{q}$ is called a $q$-ary 1 -perfect. It is known that the $q$-ary 1-perfect codes of length $n$ exist only if $n=\left(q^{m}-1\right) /(q-1)$, where $m$ is a natural number not less than two.

A mapping $\mathbb{F}_{q} \rightarrow \mathbb{F}_{q}$ is called an isometry, if it preserves distances. It is known that two codes $\mathcal{C}_{1}, \mathcal{C}_{2} \subseteq \mathbb{F}_{q}^{n}$ are isometric if and only if there are $n$ permutations $\sigma_{1}, \sigma_{2}, \ldots, \sigma_{n}$ of the elements in $\mathbb{F}_{q}$ and permutation $\pi$ of the coordinates such that $\mathcal{C}_{2}=\left\{\pi\left(\sigma_{1}\left(c_{1}\right), \sigma_{2}\left(c_{2}\right), \ldots, \sigma_{n}\left(c_{n}\right)\right):\left(c_{1}, c_{2}, \ldots, c_{n}\right) \in \mathcal{C}_{1}\right\}$.

Two codes $\mathcal{C}_{1}, \mathcal{C}_{2} \subseteq \mathbb{F}_{q}^{n}$ to be equivalent if there exists a vector $\mathbf{v} \in \mathbb{F}_{q}^{n}$ and $n \times n$ monomial matrix $M$ over $\mathbb{F}_{q}$ such that $\mathcal{C}_{2}=\left\{(\mathbf{v}+\mathbf{c} M): \mathbf{c} \in \mathcal{C}_{1}\right\}$.

In binary case, two codes are equivalent if and only if they are isometric. In nonbinary case, if $q \geq 5$ then a linear $q$-ary 1-perfect code can be isometric to a nonlinear $q$-ary 1-perfect code.

A linear $q$-ary 1-perfect code of length $n$ is unique up to equivalence and is called a $q$-ary Hamming code. We will denote the $q$-ary Hamming code of length $n=\left(q^{m}-1\right) /(q-1)$ by $\mathcal{H}_{q, m}$.

Besides the Hamming codes, there are also linear perfect codes that are called the Golay codes. The Golay codes are unique up to equivalence. The Nonlinear perfect codes with parameters of the Golay codes do not exist. The Golay codes are listed in Table I.

The trivial perfect codes include codes that have only one codeword, codes that are the whole of $\mathbb{F}_{q}^{n}$ and binary repetition codes of odd length that consisting of only two codewords, namely, the all one vector and the all zero vector.

It is known [7], [24], [27] that any non-trivial perfect code over the finite field has the parameters of a Hamming code or a Golay code.

The rank of a code $\mathcal{C}$ is the maximum number of linearly independent codewords of $\mathcal{C}$. A code of length $n$ that has rank $n$ is said to have full rank; otherwise, the code is non-full rank.

It is known [6], [23], [25] that there exist at least $q^{q^{c n}}$ (pairwise) nonequivalent $q$-ary 1-perfect codes of length $n$ where $c=\frac{1}{q}-\epsilon$. If $q=2$ and $n=15$, then there exist exactly 5983 nonequivalent binary 1-perfect codes of length 15 , see [8].

In this paper, we propose a construction of full-rank $q$-ary 1-perfect codes over finite fields. This construction is a generalization of the Etzion and Vardy construction of full-rank binary 1-perfect codes [1]. Properties of $i$-components of $q$-ary Hamming codes are investigated and the construction of fullrank $q$-ary 1-perfect codes is based on these properties. The switching construction (Vasil'ev [25], Etzion and Vardy [1]) of 1-perfect codes are generalized for the $q$-ary 1-perfect codes. 
TABLE I

GOLAY CODES

\begin{tabular}{c|c|c|c|l}
$q$ & $n$ & $|\mathcal{C}|$ & $d(\mathcal{C})$ & name of code $\mathcal{C}$ \\
\hline 2 & 23 & 4096 & 7 & binary Golay code \\
2 & 24 & 4096 & 8 & extended binary Golay code \\
3 & 11 & 729 & 5 & ternary Golay code \\
3 & 12 & 729 & 6 & extended ternary Golay code
\end{tabular}

We give a generalization of the concept of $i$-component of 1perfect codes and introduce the concept of $(i, \sigma)$-components of 1-perfect $q$-ary codes. We also present a generalization of the Lindström [6] and Schönheim [23] construction of $q$-ary 1-perfect codes and provide a lower bound on the number of (pairwise distinct) $q$-ary 1-perfect codes of length $n$.

In Section II we present the construction of Lindström [6] and Schönheim [23]. In Section III we give a generalization of the concept of $i$-component and introduce the concept of $(i, \sigma)$-components. In Section III we also present the generalization of the switching construction. In Section IV we investigate properties of $i$-components of $q$-ary Hamming codes, we also present a generalization of the Lindström [6] and Schönheim [23] construction and provide a lower bound on the number of $q$-ary 1-perfect codes of length $n$. Section $\mathrm{V}$ presents the construction of full-rank $q$-ary 1-perfect codes over finite fields. We close in Section VI with some remarks.

\section{THE $(\mathbf{u} \mid \mathbf{u}+\mathbf{v})$ CONSTRUCTION AND GENERALIZATIONS}

In this section, we present the construction of Lindström [6] and Schönheim [23] of $q$-ary 1-perfect codes. We will start from the well-known $(\mathbf{u} \mid \mathbf{u}+\mathbf{v})$ construction.

Two codes of the same length can be combined to form a third code of twice the length. Let $\mathcal{C}_{i}$ be an $\left[n, k_{i}, d_{i}\right]_{q}$ code for $i \in\{1,2\}$, both over the same finite field $\mathbb{F}_{q}$. The $(\mathbf{u} \mid \mathbf{u}+\mathbf{v})$ construction produces the $\left[2 n, k_{1}+k_{2}, \min \left\{2 d_{1}, d_{2}\right\}\right]_{q}$ code

$$
\mathcal{C}=\left\{(\mathbf{u} \mid \mathbf{u}+\mathbf{v}): \mathbf{u} \in \mathcal{C}_{1}, \mathbf{v} \in \mathcal{C}_{2}\right\} .
$$

Let $\widehat{\mathcal{H}}_{2, m}$ be a extended binary Hamming code of length $2^{m}=n+1$. Then

$$
\widehat{\mathcal{H}}_{2, m+1}=\left\{(\mathbf{u} \mid \mathbf{u}+\mathbf{v}): \mathbf{u} \in \mathbb{F}_{2}^{n+1}, p(\mathbf{u})=0, \mathbf{v} \in \widehat{\mathcal{H}}_{2, m}\right\}
$$

is the extended binary Hamming code of length $2^{m+1}=2 n+$ 2. Let $\mathcal{H}_{2, m}$ be a binary Hamming code of length $2^{m}-1=n$. Then

$$
\mathcal{H}_{2, m+1}=\left\{(\mathbf{u}|\mathbf{u}+\mathbf{v}| p(\mathbf{u})): \mathbf{u} \in \mathbb{F}_{2}^{n}, \mathbf{v} \in \mathcal{H}_{2, m}\right\}
$$

is the binary Hamming code of length $2^{m+1}-1=2 n+1$.

Construction 1 ( Vasil'ev [25]): Given a binary 1-perfect code $\mathcal{C}_{1} \subset \mathbb{F}_{2}^{n}$ and function $\lambda$ defined on $\mathcal{C}_{1}$ with values in $\mathbb{F}_{2}$, we construct the code $\mathcal{C} \subset \mathbb{F}_{2}^{2 n+1}$. Let

$$
\mathcal{C}=\left\{(\mathbf{u}|\mathbf{u}+\mathbf{v}| p(\mathbf{u})+\lambda(\mathbf{v})): \mathbf{u} \in \mathbb{F}_{2}^{n}, \mathbf{v} \in \mathcal{C}_{1}\right\} .
$$

The code $\mathcal{C}$ is a binary 1-perfect code of length $2 n+1$. If function $\lambda$ is nonlinear then the 1-perfect code $\mathcal{C}$ is nonlinear.

Next, we describe so called doubling construction of binary 1-perfect codes (or extended binary 1-perfect codes). The doubling construction is a combinatorial generalization of the $(\mathbf{u} \mid \mathbf{u}+\mathbf{v})$ construction for the binary 1-perfect codes.

First we give definition of a map $\varphi$. Let $\mathcal{C}_{0}, \mathcal{C}_{1}, \ldots, \mathcal{C}_{n}$ be a partition of $\mathbb{F}_{2}^{n}$ into binary 1-perfect codes of length $n$. We denote by $Q$ the set $\{0,1, \ldots, n\}$ and define a map $\varphi: \mathbb{F}_{2}^{n} \rightarrow$ $Q$. If $\mathbf{u} \in \mathcal{C}_{k}$, then $\varphi(\mathbf{u})=k$, where $k \in Q$.

\section{Construction 2 (Doubling Construction): Let}

$\mathcal{C}_{0}^{1}, \mathcal{C}_{1}^{1}, \ldots, \mathcal{C}_{n}^{1}$ and $\mathcal{C}_{0}^{2}, \mathcal{C}_{1}^{2}, \ldots, \mathcal{C}_{n}^{2}$ be two partitions of $\mathbb{F}_{2}^{n}$ into binary 1-perfect codes of length $n$ and $\pi: Q \rightarrow Q$ be a permutation of the set $Q$. Then

$$
\begin{aligned}
\mathcal{C}= & \{(\mathbf{u}|\mathbf{v}| p(\mathbf{u})): \\
& \left.\mathbf{u} \in \mathbb{F}_{q}^{n}=\mathcal{C}_{0}^{1} \cup \mathcal{C}_{1}^{1} \cup \cdots \cup \mathcal{C}_{n}^{1} \text { and } \mathbf{v} \in \mathcal{C}_{\pi(\varphi(\mathbf{u}))}^{2}\right\} .
\end{aligned}
$$

It is obvious, that $\mathcal{C}$ is a binary 1-perfect code of length $2 n+1$.

Heden [3] constructed by the doubling construction a nonlinear binary 1-perfect code of length $n=15$ that is not equivalent to the Vasil'ev codes. Phelps [10] and Solov'eva [22] constructed partitions of $\mathbb{F}_{2}^{n}$ into binary 1-perfect codes of length $n$, that is not partitions into translations of a 1-perfect code or partitions into cosets of a Hamming code.

Lindström [6] and Schönheim [23] have generalized the construction of Vasil'ev to the $q$-ary 1-perfect codes.

Construction 3 (Lindström [6], Schönheim [23]): Given a $q$-ary 1-perfect code $\mathcal{C}_{1} \subset \mathbb{F}_{q}^{n}$ and function $\lambda$ defined on $\mathcal{C}_{1}$ with values in $\mathbb{F}_{q}$ we construct the code $\mathcal{C} \subset \mathbb{F}_{q}^{q n+1}$. Let $\alpha_{1}, \alpha_{2}, \ldots, \alpha_{q-1}$ be all nonzero elements in $\mathbb{F}_{q}$. Then

$$
\begin{aligned}
\mathcal{C}=\{ & \left(\mathbf{u}_{1}\left|\mathbf{u}_{2}\right| \cdots\left|\mathbf{u}_{q-1}\right| \mathbf{v}+\sum_{i=1}^{q-1} \mathbf{u}_{i} \mid \sum_{i=1}^{q-1} \alpha_{i} p\left(\mathbf{u}_{i}\right)+\lambda(\mathbf{v})\right): \\
& \left.\mathbf{u}_{i} \in \mathbb{F}_{q}^{n}, \alpha_{i} \in \mathbb{F}_{q} \backslash\{0\}, i \in\{1, \ldots, q-1\}, \mathbf{v} \in \mathcal{C}_{1}\right\} .
\end{aligned}
$$

The code $\mathcal{C}$ is a $q$-ary 1-perfect code of length $q n+1$.

\section{The $(i, \sigma)$-COMPONENTS}

In this section, we give a generalization of the concept of $i$-component and introduce the concept of $(i, \sigma)$-components and we also present the generalization of switching construction.

Let $\mathcal{C}$ be a code over $\mathbb{F}_{q}$. A distance graph of the code $\mathcal{C}$ is a graph whose vertex set is $\mathcal{C}$ and vertices $\mathbf{x}, \mathbf{y} \in \mathcal{C}$ are adjacent if and only if $d(\mathbf{x}, \mathbf{y})=d$, where $d$ is a fixed natural number. If $d$ is minimum distance of the code $\mathcal{C}$, then the distance graph is called minimum distance graph. We can puncture $\mathcal{C}$ by deleting the same coordinate $i$ in each codeword. We denote the punctured code by $\mathcal{C}^{i}$.

Next, we give the Vasil'ev definition of an $i$-component of binary 1-perfect code $\mathcal{C}$ of length $n$, where a coordinate $i \in\{1,2, \ldots, n\}$.

Definition 1 (Vasil'ev [26]): An $i$-component of a $\operatorname{code} \mathcal{C}$ is a subcode (or subset) of the $\mathcal{C}$ and codewords of $i$-component of $\mathcal{C}$ correspond to vertices of connected component of minimum distance graph of the punctured code $\mathcal{C}^{i}$. The set of all $i$-components of $\mathcal{C}$ correspond to the set of all connected components of minimum distance graph of the punctured code $\mathcal{C}^{i}$. 
Example 1: Consider the binary Hamming code

$\mathcal{H}_{2,3}=\left\{(\mathbf{u}|\mathbf{u}+\mathbf{v}| p(\mathbf{u})): \mathbf{u} \in \mathbb{F}_{2}^{3}, \mathbf{v} \in\{(0,0,0),(1,1,1)\}\right\}$

of length 7 . Suppose that coordinate $i=7$. Then, the binary Hamming code $\mathcal{H}_{2,3}$ has two 7 -components:

$$
\begin{aligned}
\mathcal{R}_{7}=\left\{\begin{array}{l}
(0,0,0,0,0,0,0) \\
(0,0,1,0,0,1,1) \\
(0,1,0,0,1,0,1) \\
(0,1,1,0,1,1,0) \\
(1,0,0,1,0,0,1) \\
(1,0,1,1,0,1,0) \\
(1,1,0,1,1,0,0) \\
(1,1,1,1,1,1,1)
\end{array}\right\}, \\
\mathcal{R}_{7}+(0,0,0,1,1,1,0)=\left\{\begin{array}{l}
(0,0,0,1,1,1,0) \\
(0,0,1,1,1,0,1) \\
(0,1,0,1,0,1,1) \\
(0,1,1,1,0,0,0) \\
(1,0,0,0,1,1,1) \\
(1,0,1,0,1,0,0) \\
(1,1,0,0,0,1,0) \\
(1,1,1,0,0,0,1)
\end{array}\right\} .
\end{aligned}
$$

A binary Hamming code of length 7 has two $i$-components for each coordinate $i \in\{1,2, \ldots, 7\}$.

It is not difficult to generalize the definition of Vasil'ev to $q$-ary 1-perfect codes. Further we give a generalization of the switching construction for arbitrary $q$-ary 1-perfect codes.

Given an $i$-component $\mathcal{R}_{i} \subseteq \mathbb{F}_{q}^{n}$ and permutation $\sigma$ of the elements in $\mathbb{F}_{q}$, we define the code $\sigma\left(\mathcal{R}_{i}\right)$. Let

$$
\begin{aligned}
\sigma\left(\mathcal{R}_{i}\right)=\left\{\left(x_{1}, x_{2}, \ldots,\right.\right. & \left.\sigma\left(x_{i}\right), \ldots, x_{n}\right): \\
& \left.\left(x_{1}, x_{2}, \ldots, x_{i}, \ldots, x_{n}\right) \in \mathcal{R}_{i}\right\} .
\end{aligned}
$$

Construction 4: Let $\left\{\mathcal{R}_{i}(1), \mathcal{R}_{i}(2), \ldots, \mathcal{R}_{i}(t)\right\}$ be a family of $i$-components of a $q$-ary 1-perfect code $\mathcal{C}_{1}$ of length $n$ and let $\sigma_{1}, \sigma_{2}, \ldots, \sigma_{t}$ be $t$ permutations of the elements in $\mathbb{F}_{q}$. Then we construct the code $\mathcal{C}$. Let

$$
\mathcal{C}=\left(\mathcal{C}_{1} \backslash \bigcup_{s=1}^{t} \mathcal{R}_{i}(s)\right) \bigcup\left(\bigcup_{s=1}^{t} \sigma_{s}\left(\mathcal{R}_{i}(s)\right)\right) .
$$

Theorem 1: The code $\mathcal{C}$ is a $q$-ary 1-perfect code of length $n$.

\section{Proof:}

We need to prove that the number of codewords in the code $\mathcal{C}$ is correct and that the minimum distance $d(\mathcal{C})$ of the code $\mathcal{C}$ is equal to 3 . Obviously that $\left|\mathcal{R}_{i}(s)\right|=\left|\sigma_{s}\left(\mathcal{R}_{i}(s)\right)\right|$. By definition of $i$-components, it follows that the $i$-components from $\left\{\mathcal{R}_{i}(1), \mathcal{R}_{i}(2), \ldots, \mathcal{R}_{i}(t)\right\}$ pairwise disjoint. Thus we have

$$
|\mathcal{C}|=\left|\mathcal{C}_{1}\right|-\sum_{s=1}^{t}\left|\mathcal{R}_{i}(s)\right|+\sum_{s=1}^{t}\left|\sigma_{s}\left(\mathcal{R}_{i}(s)\right)\right|=\left|\mathcal{C}_{1}\right|=q^{n-m} .
$$

The permutation $\sigma_{s}$ defines an isometric transformation on the space $\mathbb{F}_{q}^{n}$. Hence we have $d\left(\sigma_{s}\left(\mathcal{R}_{i}(s)\right)\right)=3, s \in\{1,2, \ldots, t\}$. Consider punctured codes $\left(\mathcal{R}_{i}(s)\right)^{i}$ and $\left(\mathcal{C}_{1} \backslash \mathcal{R}_{i}(s)\right)^{i}$. It follows from the definition of the $i$-components of the code that for any $\mathbf{x} \in\left(\mathcal{R}_{i}(s)\right)^{i}$ and for any $\mathbf{y} \in\left(\mathcal{C}_{1} \backslash \mathcal{R}_{i}(s)\right)^{i}$ there holds the inequality $d(\mathbf{x}, \mathbf{y}) \geq 3$. Hence for any $\mathbf{x} \in \sigma_{s}\left(\mathcal{R}_{i}(s)\right)$ and for any $\mathbf{y} \in\left(\mathcal{C}_{1} \backslash \mathcal{R}_{i}(s)\right)$ we have $d(\mathbf{x}, \mathbf{y}) \geq 3$. Since the $i$-components from $\left\{\mathcal{R}_{i}(1), \mathcal{R}_{i}(2), \ldots, \mathcal{R}_{i}(t)\right\}$ pairwise disjoint, we obtain that the code $\mathcal{C}$ is a $q$-ary 1-perfect code of length $n$.

In binary case, there exists a unique nontrivial permutation of the elements in $\mathbb{F}_{2}$ and formula (2) takes the form

$$
\mathcal{C}=\left(\mathcal{C}_{1} \backslash \bigcup_{s=1}^{t} \mathcal{R}_{i_{s}}\right) \bigcup\left(\bigcup_{s=1}^{t} \mathcal{R}_{i_{s}}+\mathbf{e}_{i_{s}}\right)
$$

where $\mathbf{e}_{i_{s}}$ is a vector in which $i_{s}$-th component is equal to 1 and other components are equal to 0 .

We now give a generalization of the concept of $i$-component of 1-perfect codes and introduce the concept of $(i, \sigma)$ components of $q$-ary 1-perfect codes. Particular cases of the $(i, \sigma)$-components were considered in [15], [14]. We present two definition of $(i, \sigma)$-components. Following [15], we give a recursive definition of the $(i, \sigma)$-components.

Definition 2: Given a $q$-ary 1-perfect code $\mathcal{C} \subset \mathbb{F}_{q}^{n}$, coordinate $i$, and permutation $\sigma$ of the elements in $\mathbb{F}_{q}$, a subcode $\mathcal{R}_{(i, \sigma)} \subseteq \mathcal{C}$ is an $(i, \sigma)$-component of the code $\mathcal{C}$ if $\mathbf{y} \in \mathcal{R}_{(i, \sigma)}$ implies that

$$
\{\mathbf{x}: \mathbf{x} \in \mathcal{C}, d(\mathbf{x}, \mathbf{y}(i, \sigma))=2\} \subseteq \mathcal{R}_{(i, \sigma)}
$$

where

$$
\mathbf{y}(i, \sigma)=\left(y_{1}, y_{2}, \ldots, \sigma\left(y_{i}\right), \ldots, y_{n}\right) .
$$

Following [14], we formulate the definition of the $(i, \sigma)$ components in terms of graph theory. Given a $q$-ary code $\mathcal{C} \subseteq$ $\mathbb{F}_{q}^{n}$, coordinate $i$ and permutation $\sigma$ of the elements in $\mathbb{F}_{q}$, we define the code $\mathcal{C}(i, \sigma)$. Let

$$
\begin{aligned}
\mathcal{C}(i, \sigma)=\left\{\left(x_{1}, x_{2}, \ldots,\right.\right. & \left.\sigma\left(x_{i}\right), \ldots, x_{n}\right): \\
& \left.\left(x_{1}, x_{2}, \ldots, x_{i}, \ldots, x_{n}\right) \in \mathcal{C}\right\} .
\end{aligned}
$$

Definition 3: Given a $q$-ary 1-perfect code $\mathcal{C} \subset \mathbb{F}_{q}^{n}$, coordinate $i$, and permutation $\sigma$ of the elements in $\mathbb{F}_{q}$, we consider the distance bipartite graph of the code $\mathcal{C} \cup \mathcal{C}(i, \sigma)$. Two codewords $\mathbf{x} \in \mathcal{C}$ and $\mathbf{y} \in \mathcal{C}(i, \sigma)$ are adjacent if and only if $d(\mathbf{x}, \mathbf{y})=2$. The $(i, \sigma)$-components of the code $\mathcal{C}$ correspond to the connected components of the distance bipartite graph of the code $\mathcal{C} \cup \mathcal{C}(i, \sigma)$.

Let $\mathcal{R}_{i, \sigma}$ be an $(i, \sigma)$-component of a $q$-ary 1-perfect code $\mathcal{C}_{1}$ of length $n$ and let

$$
\begin{aligned}
\sigma\left(\mathcal{R}_{i, \sigma}\right)=\left\{\left(x_{1}, x_{2}, \ldots,\right.\right. & \left.\sigma\left(x_{i}\right), \ldots, x_{n}\right): \\
& \left.\left(x_{1}, x_{2}, \ldots, x_{i}, \ldots, x_{n}\right) \in \mathcal{R}_{i, \sigma}\right\} .
\end{aligned}
$$

Then, it is obvious that

$$
\mathcal{C}=\left(\mathcal{C}_{1} \backslash \mathcal{R}_{i, \sigma}\right) \cup\left(\sigma\left(\mathcal{R}_{i, \sigma}\right)\right)
$$

is a $q$-ary 1-perfect code of length $n$.

In binary case, an $(i, \sigma)$-component is an $i$-component. In nonbinary case, $(i, \sigma)$-components form a partition of an $i$ component. 
Let $\mathcal{C}_{1}$ be a $q$-ary 1-perfect code, $\mathcal{R}_{i, \sigma}$ be an $(i, \sigma)$ component of $\mathcal{C}_{1}$ and let

$$
\mathcal{C}_{2}=\left(\mathcal{C}_{1} \backslash \mathcal{R}_{i, \sigma}\right) \cup\left(\sigma\left(\mathcal{R}_{i, \sigma}\right)\right) .
$$

Then we say that the code $\mathcal{C}_{2}$ is obtained from $\mathcal{C}_{1}$ by a switching $(i, \sigma)$-component $\mathcal{R}_{i, \sigma}$.

Let $\mathcal{C}_{1}, \mathcal{C}_{2}, \ldots, \mathcal{C}_{t}$ be the $q$-ary 1-perfect codes of length $n$ and let code $C_{s+1}$ be obtained from $C_{s}$ by a switching $\left(i_{s}, \sigma_{s}\right)$ component, where $s \in\{1,2, \ldots, t-1\}, i_{s} \in\{1,2, \ldots, n\}$, $\sigma_{s}$ is a permutation of the elements in $\mathbb{F}_{q}$. Then we say that $\mathcal{C}_{t}$ is obtained from $\mathcal{C}_{1}$ by a sequence of switchings.

The switching class of a $q$-ary 1-perfect code $\mathcal{C}$ is a collection of all nonequivalent $q$-ary 1-perfect codes that can be obtained from $\mathcal{C}$ by a sequence of switchings.

A full-rank $q$-ary 1-perfect code is called Type I, if its switching class contains non-full-rank codes; otherwise, the code is called Type II.

The problem of existence of Type II full-rank $q$-ary 1-perfect codes is open. Originally, this problem was posed for binary codes in [19].

It is known [9] that there are 9 switching classes for the binary 1-perfect codes of length 15 , and their sizes are 5819 , $153,3,2,2,1,1,1$, and 1 . The switching class of the Hamming code have 5819 nonequivalent codes and in fact contains all codes with full rank except two. The two full-rank codes that are not in the switching class of the Hamming code have one more code in their switching class, a code with rank 14 . Consequently, the Type II full-rank binary 1-perfect codes of length 15 do not exist.

Phelps and LeVan [11] constructed, by the doubling construction, a binary 1-perfect code of length 15 whose switching class consists of just two nonequivalent codes. Etzion and Vardy [1] showed that full-rank binary 1-perfect codes can not be constructed by doubling construction. Heden and Krotov [4] showed that non-full-rank $q$-ary 1-perfect codes have certain structural properties.

\section{PRoperties of $i$-COMPONENTS}

In this section, we investigate properties of $i$-components of $q$-ary Hamming codes, we also present a generalization of the Lindström [6] and Schönheim [23] construction and provide a lower bound on the number of $q$-ary 1-perfect codes of length $n$. At first we prove theorems describing some properties of $i$-components of $q$-ary Hamming code $\mathcal{H}_{q, m}$.

The parity-check matrix $H=\left[\mathbf{h}_{1}, \mathbf{h}_{2}, \ldots, \mathbf{h}_{n}\right]$ of the code $\mathcal{H}_{q, m}$ of length $n=\left(q^{m}-1\right) /(q-1)$ consists of $n$ pairwise linearly independent column vectors $\mathbf{h}_{i}, i \in\{1, \ldots, n\}$. The transposed column vector $\mathbf{h}_{i}^{T}$ belongs to $\mathbb{F}_{q}^{m}, i \in\{1, \ldots, n\}$. We assume that the columns of the parity-check matrix $H$ are arranged in some fixed order. The set $\mathbb{F}_{q}^{m} \backslash\{\mathbf{0}\}$ generates a projective geometry $P G_{m-1}(q)$ of dimension $(m-1)$ over the finite field $\mathbb{F}_{q}$. In this geometry, points correspond to the columns of the parity-check matrix $H$ and the three points $i, j, k$ lie on the same line if the corresponding columns $\mathbf{h}_{\mathbf{i}}, \mathbf{h}_{\mathbf{j}}, \mathbf{h}_{\mathbf{k}}$ are linearly dependent. We denote by $l_{x y}$ the line passing through the points $x$ and $y$, and we denote by $P_{x y z}$ the plane spanned by three non-collinear points $x, y, z$. Let $\mathbf{x}=\left(x_{1}, x_{2}, \ldots, x_{n}\right) \in \mathbb{F}_{q}^{n}$, then the support of the vector $\mathbf{x}$ is the set $\operatorname{supp}(\mathbf{x})=\left\{i: x_{i} \neq 0\right\}$. A triple belongs to a line $l$ if the support of this triple belongs to the line $l$. The triples intersect at a point $i$ if their supports intersect at the point $i$.

A vector of weight 3 of the $q$-ary Hamming code $\mathcal{H}_{q, m}$ is called triple. Following [12], [13], we denote by $\mathcal{R}_{i}$ a subspace spanned by the set of all triples of the code $\mathcal{H}_{q, m}$ having 1 in the $i$-th coordinate.

Consider a vector $\mathbf{x} \in \mathbb{F}_{q}^{n}$ such that its $\operatorname{supp}(\mathbf{x})$ is $m-2$ dimensional hyperplane. Denote by $\mathbb{F}_{q}^{n}(\mathbf{x})$ the set of all vectors $\mathbf{u} \in \mathbb{F}_{q}^{n}$ such that $\operatorname{supp}(\mathbf{u}) \subseteq \operatorname{supp}(\mathbf{x})$.

Denote by $\mathcal{H}_{l}$ the subcode of $\mathcal{H}_{q, m}$ defined by a line $l$. We remind that, by definition,

$$
\mathcal{H}_{l}=\left\{\mathbf{u}: \mathbf{u} \in \mathcal{H}_{q, m} \text { and } \operatorname{supp}(\mathbf{u}) \subseteq l\right\} .
$$

Lemma 1 (Romanov [21], Lemma 1): Let $i \notin \operatorname{supp}(\mathbf{x})$ and $\mathbf{u} \in \mathbb{F}_{q}^{n}(\mathbf{x})$. Then, the intersection

$$
\left(\mathcal{R}_{i}+\mathbf{u}\right) \cap \mathbb{F}_{q}^{n}(\mathbf{x})
$$

contains only one vector.

Proof: Consider a pencil of lines $l_{1}, l_{2}, \ldots, l_{(n-1) / q}$ which pass through the point $i$. It is known [18] that

$$
\mathcal{R}_{i}=\mathcal{H}_{l_{1}}+\mathcal{H}_{l_{2}}+\cdots+\mathcal{H}_{l_{(n-1) / q}} .
$$

Let $l_{s}$ be an arbitrary line through the point $i$, where $s \in$ $\{1,2, \ldots,(n-1) / q\}$. Since $i \notin \operatorname{supp}(\mathbf{x})$, it follows that any line passing through the point $i$ intersects with the hyperplane $\operatorname{supp}(\mathbf{x})$ only at one point. We can consider projective geometry $P G_{m-1}(q)$ as the set of all subspaces of the vector space $\mathbb{F}_{q}^{n}$. A point in projective geometry is a subspace of dimension one. Hence the intersection of $\mathcal{H}_{l_{s}} \cap \mathbb{F}_{q}^{n}(\mathbf{x})$ can contain only vectors of weight 0 or 1 . It is obvious that $\mathbf{0} \in \mathcal{H}_{l_{s}} \cap \mathbb{F}_{q}^{n}(\mathbf{x})$. Since the minimum weight of the nonzero vectors in $\mathcal{H}_{l_{s}}$ is equal to 3, it follows that $\mathcal{H}_{l_{s}} \cap \mathbb{F}_{q}^{n}(\mathbf{x})=\{\mathbf{0}\}$. Since the line $l_{s}$ was chosen arbitrarily and $\mathcal{R}_{i}^{n}$ is a subspace, we get that $\mathcal{R}_{i}^{n} \cap \mathbb{F}_{q}^{n}(\mathbf{x})=\{\mathbf{0}\}$.

Each line through the point $i$ contains $q-1$ linearly independent triples. Therefore from (3) we obtain that the dimension of $\mathcal{R}_{i}$ is equal to $(q-1)((n-1) / q)=q^{m-1}-1$ see [13].

Theorem 2: Given a coordinate $i$ and $\mathbf{u} \in \mathcal{H}_{q, m}$, a code $\mathcal{R}_{i}+\mathbf{u}$ is an $i$-component of the $q$-ary Hamming code $\mathcal{H}_{q, m}$ of length $n=\left(q^{m}-1\right) /(q-1), m \geq 2$.

Proof: Consider punctured code $\mathcal{R}_{i}^{i}$. Obviously that code $\mathcal{R}_{i}^{i}$ is linear and spanned by codewords of weight 2 . Thus the code $\mathcal{R}_{i}^{i}$ is Hamiltonian and minimum distance graph of the code $\mathcal{R}_{i}^{i}$ is connected, see [21]. Without loss of generality we can assume that $i \notin \operatorname{supp}(\mathbf{x})$, where $\mathbf{x} \in \mathbb{F}_{q}^{n}$ and $\operatorname{supp}(\mathbf{x})$ is a $m-2$ dimensional hyperplane. Hence taking into account Lemma 1 we obtain that

$$
\mathcal{H}_{q, m}=\bigcup_{\mathbf{u} \in \mathcal{H}_{\mathbf{x}}} \mathcal{R}_{i}+\mathbf{u}
$$

where $\mathcal{H}_{\mathbf{x}}$ is a subcode of $\mathcal{H}_{q, m}$ defined by a $m-2$ dimensional hyperplane $\operatorname{supp}(\mathbf{x})$. Therefore, minimum distance graph of the punctured code $\left(\mathcal{R}_{i}+\mathbf{u}\right)^{i}$ is a connected component of the minimum distance graph of the punctured Hamming code $\mathcal{H}_{q, m}^{i}$. 
Following [19], we will call a subspace $\mathcal{R}_{i}$ of Hamming code $\mathcal{H}_{q, m}$ a principal $i$-component.

We now present a generalization of the Lindström [6] and Schönheim [23] construction of $q$-ary 1-perfect codes.

Construction 5: Let $\mathcal{C}_{1}$ be a $q$-ary 1-perfect code of length $n=\left(q^{m}-1\right) /(q-1), m \geq 2$, let $\mathcal{R}_{i}$ be a principal $i$ component of the $q$-ary Hamming code $\mathcal{H}_{q, m+1}, i \leq(q-$ 1) $n+1$, and let $\sigma_{\mathbf{c}}$ be a permutation of the elements in $\mathbb{F}_{q}$, $\mathbf{c} \in \mathcal{C}_{1}$. Then we construct the code $\mathcal{C}$. Let

$$
\mathcal{C}=\bigcup_{\mathbf{c} \in \mathcal{C}_{1}} \sigma_{\mathbf{c}}\left(\mathcal{R}_{i}+(\mathbf{0} \mid \mathbf{c})\right)
$$

where the zero vector $\mathbf{0} \in \mathbb{F}_{q}^{(q-1) n+1}$.

Theorem 3: The code $\mathcal{C}$ is a $q$-ary 1-perfect code of length $q n+1$.

Proof:

Consider a vector $\mathbf{x} \in \mathbb{F}_{q}^{n}$ such that its $\operatorname{supp}(\mathbf{x})$ is $m-2$ dimensional hyperplane. Without loss of generality we can assume that

$$
\operatorname{supp}(\mathbf{x})=\{(q-1) n+2,(q-1) n+3, \ldots, q n+1\} .
$$

Since $i \leq(q-1) n+1$, it follows that $i \notin \operatorname{supp}(\mathbf{x})$ Hence taking into account that $\mathcal{R}_{i}$ is the principal $i$-component of the $q$-ary Hamming code and $i \notin s u p p(\mathbf{x})$, we have that minimum distance graph of the code $\left(\mathcal{R}_{i}+(\mathbf{0} \mid \mathbf{c})\right)^{i}$ is connected. Lemma 1 implies that

$$
\left(\mathcal{R}_{i}+\left(\mathbf{0} \mid \mathbf{c}_{1}\right)\right) \cap\left(\mathcal{R}_{i}+\left(\mathbf{0} \mid \mathbf{c}_{2}\right)\right)=\varnothing
$$

for all $\mathbf{c}_{1}, \mathbf{c}_{2} \in \mathcal{C}_{1}, \mathbf{c}_{1} \neq \mathbf{c}_{2}$. Further, we consider punctured codes $\left(\mathcal{R}_{i}+\left(\mathbf{0} \mid \mathbf{c}_{1}\right)\right)^{i}$ and $\left(\mathcal{R}_{i}+\left(\mathbf{0} \mid \mathbf{c}_{2}\right)\right)^{i}$. Since $i \notin \operatorname{supp}(\mathbf{x})$, from Lemma 1 it follows that for any $\mathbf{u} \in\left(\mathcal{R}_{i}+\left(\mathbf{0} \mid \mathbf{c}_{1}\right)\right)^{i}$ and for any $\mathbf{v} \in\left(\mathcal{R}_{i}+\left(\mathbf{0} \mid \mathbf{c}_{2}\right)\right)^{i}$ there holds the inequality $d(\mathbf{u}, \mathbf{v}) \geq 3$. Hence the code $\mathcal{R}_{i}+(\mathbf{0} \mid \mathbf{c})$ is $i$-component of the code $\mathcal{C}$ for all $\mathbf{c} \in \mathcal{C}_{1}$. The set of all $i$-components $\mathcal{R}_{i}+(\mathbf{0} \mid \mathbf{c})$ form a partition of the code $\mathcal{C}$ and formula (2) takes the form (4). The dimension of $\mathcal{R}_{i}$ is $q^{m}-1$. Thus we have

$$
|\mathcal{C}|=\left|\mathcal{R}_{i}\right| \cdot\left|\mathcal{C}_{1}\right|=q^{q^{m}-1} \cdot q^{n-m}=q^{q n-m} .
$$

Hence the number of codewords in the code $\mathcal{C}$ is correct. Therefore by Theorem 1, we obtain that the code $\mathcal{C}$ is a $q$ ary 1-perfect code of length $q n+1$.

Denote by $N(q, n)$ the number of $q$-ary 1-perfect codes of length $n=\left(q^{m}-1\right) /(q-1)$. Then from the Lindström [6] and Schönheim [23] construction we have

$$
N(q, n) \geq(q)^{q^{\frac{n-1}{q}-m-1}} .
$$

From (4) and from definition of principal $i$-component of $q$ ary Hamming code it follows that by permutation of elements in $\mathbb{F}_{q}$ we obtain different 1-perfect codes. Hence we get that

$$
N(q, n) \geq(q !)^{q^{\frac{n-1}{q}-m-1}} .
$$

We now cite two theorems from [20] which will be needed in the next section.

Theorem 4 (Romanov [20], Theorem 1): Let a vector $\mathbf{u}=$ $\left(u_{1}, u_{2}, \ldots, u_{n}\right) \in \mathcal{R}_{i}$ and a component $u_{x}$ of the vector $\mathbf{u}$ be nonzero, $x \neq i$. Then, the line $l_{i x}$ has a point $y$ distinct from the points $i, x$ and such that component $u_{y}$ of the vector $\mathbf{u}$ is nonzero.

The next theorem follows directly from Theorem 4.

Theorem 5 (Romanov [20], Theorem 2): Let $i \neq j$, a vector $\mathbf{u}=\left(u_{1}, u_{2}, \ldots, u_{n}\right) \in\left(\mathcal{R}_{i}+\mathcal{R}_{j}\right)$, a component $u_{x}$ of the vector $\mathbf{u}$ be nonzero and the point $x$ does not lie on $l_{i j}$. Then, the plane $P_{i j x}$ has a point $y$ distinct from the points $i, j, x$ and such that component $u_{y}$ of the vector $\mathbf{u}$ is nonzero.

\section{FULL-RANK PERFECT CODES}

Etzion and Vardy [1] proposed a switching construction of the full-rank binary 1-perfect codes. They also proposed an original method to construct an admissible family of $i$ components of the binary Hamming code and their construction of the full-rank binary 1-perfect codes is based on this method. In [20], the method of Etzion and Vardy has been generalized to $q$-ary codes. In this section, we present a generalization of the Etzion and Vardy construction of the fullrank binary 1-perfect codes to 1-perfect codes over finite fields of characteristic 2. This generalization is based on results in [20]. Finally in this section, we present a modification of the Etzion and Vardy construction of the full-rank 1-perfect codes for finite fields of arbitrary characteristic.

So first we give a generalization of the switching construction (Etzion and Vardy [1]) for $q$-ary Hamming codes.

A family $\left\{\mathcal{R}_{i_{1}}+\mathbf{u}_{1}, \mathcal{R}_{i_{2}}+\mathbf{u}_{2}, \ldots, \mathcal{R}_{i_{t}}+\mathbf{u}_{t}\right\}$ of $i$ components (where $i \in\left\{i_{1}, i_{2}, \ldots, i_{t}\right\}$ ) of a $q$-ary Hamming code $\mathcal{H}_{q, m}$ is called admissible if for any $r, s \in\{1,2, \ldots, t\}$, $r \neq s$, we have $\left(\mathcal{R}_{i_{r}}+\mathbf{u}_{r}\right) \cap\left(\mathcal{R}_{i_{s}}+\mathbf{u}_{s}\right)=\varnothing$.

Construction 6: Let $\left\{\mathcal{R}_{i_{1}}+\mathbf{u}_{1}, \mathcal{R}_{i_{2}}+\mathbf{u}_{2}, \ldots, \mathcal{R}_{i_{t}}+\mathbf{u}_{t}\right\}$ be an admissible family of $i$-components of a $q$-ary Hamming code $\mathcal{H}_{q, m}$ of length $n=\left(q^{m}-1\right) /(q-1), i \in\left\{i_{1}, i_{2}, \ldots, i_{t}\right\}$ and let $\sigma_{1}, \sigma_{2}, \ldots, \sigma_{t}$ be $t$ permutations of the elements in $\mathbb{F}_{q}$. Then we construct the $\operatorname{code} \mathcal{C}$. Let

$$
\mathcal{C}=\left(\mathcal{H}_{q, m} \backslash \bigcup_{s=1}^{t} \mathcal{R}_{i_{s}}+\mathbf{u}_{s}\right) \bigcup\left(\bigcup_{s=1}^{t} \sigma_{s}\left(\mathcal{R}_{i_{s}}+\mathbf{u}_{s}\right)\right) \text {. }
$$

Theorem 6: The code $\mathcal{C}$ is a $q$-ary 1-perfect code of length $n=\left(q^{m}-1\right) /(q-1)$.

Proof:

We need to prove that the number of codewords in the code $\mathcal{C}$ is correct and minimum distance $d(\mathcal{C})$ of the code $\mathcal{C}$ is equal to 3 . Obviously that $\left|\mathcal{R}_{i_{s}}\right|=\left|\sigma_{s}\left(\mathcal{R}_{i_{s}}\right)\right|$. Since the family $\left\{\mathcal{R}_{i_{1}}+\mathbf{u}_{1}, \mathcal{R}_{i_{2}}+\mathbf{u}_{2}, \ldots, \mathcal{R}_{i_{t}}+\mathbf{u}_{t}\right\}$ of $i$-components is admissible, it follows from (5) that

$$
|\mathcal{C}|=\left|\mathcal{C}_{1}\right|-\sum_{s=1}^{t}\left|\mathcal{R}_{i_{s}}\right|+\sum_{s=1}^{t}\left|\sigma_{s}\left(\mathcal{R}_{i_{s}}\right)\right|=\left|\mathcal{C}_{1}\right|=q^{n-m} .
$$

Further, we show that $d(\mathcal{C})=3$. The permutation $\sigma_{s}$ defines an isometric transformation on the space $\mathbb{F}_{q}^{n}$. Thus we have $d\left(\sigma_{s}\left(\mathcal{R}_{i_{s}}\right)\right)=3, s \in\{1,2, \ldots, t\}$.

Consider an $i$-component $\mathcal{R}_{i}+\mathbf{u}$ and $j$-component $\mathcal{R}_{j}+\mathbf{v}$ from $\left\{\mathcal{R}_{i_{1}}+\mathbf{u}_{1}, \mathcal{R}_{i_{2}}+\mathbf{u}_{2}, \ldots, \mathcal{R}_{i_{t}}+\mathbf{u}_{t}\right\}$. We suppose that $i \neq j$. (If $i=j$ then see Theorem 1.) Without loss of generality assume that $i \notin \operatorname{supp}(\mathbf{x})$ and $j \notin \operatorname{supp}(\mathbf{x})$ where $\operatorname{supp}(\mathbf{x})$ is $m-2$ dimensional hyperplane, $\mathbf{x} \in \mathbb{F}_{q}^{n}$. From the conditions of the theorem it follows that $\left(\mathcal{R}_{i}+\mathbf{u}\right) \cap\left(\mathcal{R}_{j}+\mathbf{v}\right)=\varnothing$. Further, 
we consider punctured codes $\left(\mathcal{R}_{i}+\mathbf{u}\right)^{i}$ and $\left(\mathcal{R}_{j}+\mathbf{v}\right)^{j}$. Since $i \notin \operatorname{supp}(\mathbf{x})$ and $j \notin \operatorname{supp}(\mathbf{x})$, we obtain from Lemma 1 that for any $\mathbf{c} \in\left(\mathcal{R}_{i}+\mathbf{u}\right)^{i}$ and for any $\mathbf{c}^{\prime} \in\left(\mathcal{R}_{j}+\mathbf{v}\right)^{j}$ there holds the inequality $d\left(\mathbf{c}, \mathbf{c}^{\prime}\right) \geq 3$. Therefore $d(\mathcal{C})=3$.

Next, we construct the vectors $\mathbf{c}_{1}, \mathbf{c}_{1}, \ldots, \mathbf{c}_{m}$ and show that these vectors are the codewords of the Hamming code $\mathcal{H}_{q, m}$ of length $n=q^{m}-1 / q-1$.

In the parity-check matrix $H=\left[\mathbf{h}_{\mathbf{1}}, \mathbf{h}_{\mathbf{2}}, \ldots, \mathbf{h}_{\mathbf{n}}\right]$ of the Hamming code $\mathcal{H}_{q, m}$, we choose $m$ linearly independent columns. Without loss of generality assume that we have chosen the columns $\mathbf{h}_{\mathbf{1}}, \mathbf{h}_{\mathbf{2}}, \ldots, \mathbf{h}_{\mathbf{m}}$.

For each $\mathbf{z} \in \mathbb{F}_{q}^{m} \backslash\{\mathbf{0}\}$ there exists a unique scalar $\alpha \in \mathbb{F}_{q} \backslash$ $\{0\}$ and a unique vector column $\mathbf{h}_{i} \in H$ such that $\mathbf{z}=\alpha \mathbf{h}_{i}^{T}$. Define a mapping $\xi$ from the nonzero vectors of $\mathbb{F}_{q}^{m}$ onto the vectors of weight 1 in $\mathbb{F}_{q}^{n}$ as follows:

$$
\begin{gathered}
\forall \mathbf{z} \in \mathbb{F}_{q}^{m} \backslash\{\mathbf{0}\}, \xi(\mathbf{z})=\left(x_{1}, x_{2}, \ldots, x_{n}\right) \in \mathbb{F}_{q}^{n} \\
\text { where } x_{i}=\left\{\begin{array}{cl}
\alpha, & \text { if } \mathbf{z}=\alpha \mathbf{h}_{i}^{T}, \\
0, & \text { if } \mathbf{z} \neq \alpha \mathbf{h}_{i}^{T} .
\end{array}\right.
\end{gathered}
$$

From now on, we will use the notation $\xi\left(\mathbf{h}_{i}\right)$ instead of the notation $\xi\left(\mathbf{h}_{i}^{T}\right)$. Following [1], we define

$$
\begin{aligned}
\mathbf{c}_{1}= & \xi\left(\mathbf{h}_{1}\right)+\xi\left(\mathbf{h}_{1}+\mathbf{h}_{2}+\mathbf{h}_{3}\right)+\xi\left(\mathbf{h}_{1}+\mathbf{h}_{2}+\mathbf{h}_{4}\right) \\
& +\xi\left(\mathbf{h}_{1}+\mathbf{h}_{3}+\mathbf{h}_{4}\right), \\
\mathbf{c}_{2}= & \xi\left(\mathbf{h}_{1}\right)+\xi\left(\mathbf{h}_{2}\right)+\xi\left(\mathbf{h}_{1}+\mathbf{h}_{3}+\mathbf{h}_{4}\right) \\
& +\xi\left(\mathbf{h}_{2}+\mathbf{h}_{3}+\mathbf{h}_{4}\right), \\
\mathbf{c}_{4}= & \xi\left(\mathbf{h}_{1}\right)+\xi\left(\mathbf{h}_{2}\right)+\xi\left(\mathbf{h}_{3}\right)+\xi\left(\mathbf{h}_{4}\right) \\
& +\xi\left(\mathbf{h}_{1}+\mathbf{h}_{2}+\mathbf{h}_{3}\right)+\xi\left(\mathbf{h}_{1}+\mathbf{h}_{2}+\mathbf{h}_{4}\right) \\
& +\xi\left(\mathbf{h}_{1}+\mathbf{h}_{3}+\mathbf{h}_{4}\right)+\xi\left(\mathbf{h}_{2}+\mathbf{h}_{3}+\mathbf{h}_{4}\right) .
\end{aligned}
$$

Further let $j \in\{1,2, \ldots, m\} \backslash\{1,2,4\}$. If $j$ is odd, define

$$
\mathbf{c}_{j}=\sum_{i=1}^{j} \xi\left(\mathbf{h}_{i}\right)+\xi\left(\mathbf{h}_{1}+\mathbf{h}_{2}+\cdots+\mathbf{h}_{j}\right) .
$$

Otherwise set

$$
\begin{aligned}
\mathbf{c}_{j}=\sum_{i=1}^{j} \xi\left(\mathbf{h}_{i}\right)+ & \xi\left(\mathbf{h}_{1}+\mathbf{h}_{2}+\cdots+\mathbf{h}_{j / 2}\right) \\
& +\xi\left(\mathbf{h}_{j / 2+1}+\mathbf{h}_{j / 2+2}+\cdots+\mathbf{h}_{j}\right) .
\end{aligned}
$$

Lemma 2: If a finite field $\mathbb{F}_{q}$ has characteristic 2 and $m \geq$ 4. Then, $\mathbf{c}_{i} \in \mathcal{H}_{q, m}, i=1,2, \ldots, m$.

Proof: Since the finite field $\mathbb{F}_{q}$ has characteristic 2 it follows by construction of $\mathbf{c}_{i}$ that $\mathbf{c}_{i} \in \mathcal{H}_{q, m}, i=1,2, \ldots, m$.

Theorem 2 implies that $\mathcal{R}_{i}+\mathbf{u}$ is an $i$-component of the $q$ ary Hamming code $\mathcal{H}_{q, m}$ if $\mathbf{u} \in \mathcal{H}_{q, m}$. Therefore by Lemma 1 it is clear that the family

$$
\mathcal{F}=\left\{\mathcal{R}_{1}+\mathbf{c}_{1}, \mathcal{R}_{2}+\mathbf{c}_{2}, \ldots, \mathcal{R}_{m}+\mathbf{c}_{m}\right\}
$$

is a family of $i$-components of the Hamming code $\mathcal{H}_{q, m}$.

Further we study the properties of the family $\mathcal{F}$ of $i$ components of the Hamming code $\mathcal{H}_{q, m}$.

Lemma 3: If finite field $\mathbb{F}_{q}$ has characteristic 2 and $m \geq 4$. Then, the family $\mathcal{F}$ of $i$-components is an admissible family of $i$-components of the Hamming code $\mathcal{H}_{q, m}$ over the finite field $\mathbb{F}_{q}$.

Proof: Let $r, s \in\{1,2, \ldots, m\}, r \neq s$. Then, we show that

$$
\left(\mathcal{R}_{r}+\mathbf{c}_{r}\right) \cap\left(\mathcal{R}_{s}+\mathbf{c}_{s}\right)=\varnothing .
$$

In order to satisfy the equality 6 , it suffices to show that

$$
\mathbf{c}_{r}-\mathbf{c}_{s} \notin \mathcal{R}_{r}+\mathcal{R}_{s} .
$$

By Theorem 5, it suffices to show that the support of vector $\mathbf{c}_{r}-\mathbf{c}_{s}$ contains a point $x$, not lying on the line $l_{r s}$ and such that no other point (distinct from the points $r, s, x$ ) in the $\operatorname{supp}\left(\mathbf{c}_{r}-\mathbf{c}_{s}\right)$ does not belong to the plane $P_{r s x}$. We consider several cases.

Case 1: Let $r=1, s=2$. Then

$$
\begin{aligned}
\operatorname{supp}\left(\mathbf{c}_{1}-\mathbf{c}_{2}\right)=\{2\} \cup \operatorname{supp}( & \left.\left(\mathbf{h}_{1}+\mathbf{h}_{2}+\mathbf{h}_{3}\right)\right) \\
& \cup \operatorname{supp}\left(\xi\left(\mathbf{h}_{1}+\mathbf{h}_{2}+\mathbf{h}_{4}\right)\right) .
\end{aligned}
$$

Suppose that $x=\operatorname{supp}\left(\xi\left(\mathbf{h}_{1}+\mathbf{h}_{2}+\mathbf{h}_{3}\right)\right)$. Since the columns $\mathbf{h}_{\mathbf{1}}, \mathbf{h}_{\mathbf{2}}, \ldots, \mathbf{h}_{\mathbf{m}}$ are linearly independent it is obvious that $\left(\mathbf{h}_{1}+\mathbf{h}_{2}+\mathbf{h}_{4}\right) \notin P_{12 x}$.

The cases when $r=1$ or $r=2$ and $s=3$ or $s=4$ are proved similarly.

Case 2: Let $r, s \in\{1,2, \ldots, m\} \backslash\{1,2,4\}, r \neq s$ and $r, s$ is odd. Without loss of generality we can assume that $r<s$. Then we have

$$
\begin{aligned}
& \operatorname{supp}\left(\mathbf{c}_{r}-\mathbf{c}_{s}\right)=\{r+1, r+2, \ldots, s\} \\
& \bigcup \operatorname{supp}\left(\xi\left(\sum_{i=1}^{r} \mathbf{h}_{i}\right)\right) \bigcup \operatorname{supp}\left(\xi\left(\sum_{i=1}^{s} \mathbf{h}_{i}\right)\right) .
\end{aligned}
$$

Suppose that $x=r+1$. Then for any

$$
y \in \operatorname{supp}\left(\mathbf{c}_{r}-\mathbf{c}_{s}\right) \backslash\{r, s, r+1\}
$$

it is clear that $y \notin P_{r s r+1}$.

Case 3: Let $r, s \in\{1,2, \ldots, m\} \backslash\{1,2,4\}, r \neq s$ and $r, s$ is even. Without loss of generality we assume that $r<s$. Then we have

$$
\begin{aligned}
& \operatorname{supp}\left(\mathbf{c}_{r}-\mathbf{c}_{s}\right)=\{r+1, r+2, \ldots, s\} \\
& \bigcup \operatorname{supp}\left(\xi\left(\sum_{i=1}^{r / 2} \mathbf{h}_{i}\right)\right) \bigcup \operatorname{supp}\left(\xi\left(\sum_{i=r / 2+1}^{r} \mathbf{h}_{i}\right)\right) \\
& \bigcup \operatorname{supp}\left(\xi\left(\sum_{i=1}^{s / 2} \mathbf{h}_{i}\right)\right) \bigcup \operatorname{supp}\left(\xi\left(\sum_{i=s / 2+1}^{s} \mathbf{h}_{i}\right)\right) .
\end{aligned}
$$

Suppose that $x=\operatorname{supp}\left(\xi\left(\sum_{i=1}^{r / 2} \mathbf{h}_{i}\right)\right)$. Then for any

$$
y \in \operatorname{supp}\left(\mathbf{c}_{r}-\mathbf{c}_{s}\right) \backslash\{r, s, x\}
$$

it is clear that $y \notin P_{r s x}$.

Case 4: Let $r, s \in\{1,2, \ldots, m\} \backslash\{1,2,3,4\}, r \neq s, r$ is even and $s$ is odd. Without loss of generality we assume that $r<s$. Then we have

$$
\begin{array}{r}
\operatorname{supp}\left(\mathbf{c}_{r}-\mathbf{c}_{s}\right)=\{r+1, r+2, \ldots, s\} \\
\bigcup \operatorname{supp}\left(\xi\left(\sum_{i=1}^{r / 2} \mathbf{h}_{i}\right)\right) \bigcup \operatorname{supp}\left(\xi\left(\sum_{i=r / 2+1}^{r} \mathbf{h}_{i}\right)\right) \\
\bigcup \operatorname{supp}\left(\xi\left(\sum_{i=1}^{s} \mathbf{h}_{i}\right)\right) .
\end{array}
$$

Suppose that $x=\operatorname{supp}\left(\xi\left(\sum_{i=1}^{r / 2} \mathbf{h}_{i}\right)\right)$. Then for any

$$
y \in \operatorname{supp}\left(\mathbf{c}_{r}-\mathbf{c}_{s}\right) \backslash\{r, s, x\}
$$


it is clear that $y \notin P_{r s x}$.

Case 5: Let $r=1, s \in\{1,2, \ldots, m\} \backslash\{1,2,3,4\}$ and $s$ is odd.

$$
\begin{array}{r}
\operatorname{supp}\left(\mathbf{c}_{r}-\mathbf{c}_{s}\right)=\{2,3, \ldots, s\} \bigcup \operatorname{supp}\left(\xi\left(\mathbf{h}_{1}+\mathbf{h}_{2}+\mathbf{h}_{3}\right)\right) \\
\bigcup \operatorname{supp}\left(\xi\left(\mathbf{h}_{1}+\mathbf{h}_{2}+\mathbf{h}_{4}\right)\right) \bigcup \operatorname{supp}\left(\xi\left(\mathbf{h}_{1}+\mathbf{h}_{3}+\mathbf{h}_{4}\right)\right) \\
\bigcup \operatorname{supp}\left(\xi\left(\sum_{i=1}^{s} \mathbf{h}_{i}\right)\right) .
\end{array}
$$

Suppose that $x=s-1$. Then for any

$$
y \in \operatorname{supp}\left(\mathbf{c}_{r}-\mathbf{c}_{s}\right) \backslash\{r, s, x\}
$$

it is clear that $y \notin P_{r s x}$. The cases when $r=1,2,4$ and $s$ is odd are proved similarly.

Case 6: Let $r=1, s \in\{1,2, \ldots, m\} \backslash\{1,2,4\}$ and $s$ is even. Let $r=1, s \in\{1,2, \ldots, m\} \backslash\{1,2,3,4\}$ and $s$ is odd.

$$
\begin{gathered}
\operatorname{supp}\left(\mathbf{c}_{r}-\mathbf{c}_{s}\right)=\{2,3, \ldots, s\} \bigcup \operatorname{supp}\left(\xi\left(\mathbf{h}_{1}+\mathbf{h}_{2}+\mathbf{h}_{3}\right)\right) \\
\bigcup \operatorname{supp}\left(\xi\left(\mathbf{h}_{1}+\mathbf{h}_{2}+\mathbf{h}_{4}\right)\right) \bigcup \operatorname{supp}\left(\xi\left(\mathbf{h}_{1}+\mathbf{h}_{3}+\mathbf{h}_{4}\right)\right) \\
\bigcup \operatorname{supp}\left(\xi\left(\sum_{i=1}^{s / 2} \mathbf{h}_{i}\right)\right) \bigcup \operatorname{supp}\left(\xi\left(\sum_{i=s / 2+1}^{s} \mathbf{h}_{i}\right)\right) .
\end{gathered}
$$

Suppose that $x=s-1$. Then for any

$$
y \in \operatorname{supp}\left(\mathbf{c}_{r}-\mathbf{c}_{s}\right) \backslash\{r, s, x\}
$$

it is clear that $y \notin P_{r s x}$. The cases when $r=1,2,4$ and $s$ is even are proved similarly.

Theorem 7: Let $\mathbb{F}_{q}$ be a finite field of characteristic 2, let $\mathcal{F}=\left\{\mathcal{R}_{1}+\mathbf{c}_{1}, \mathcal{R}_{2}+\mathbf{c}_{2}, \ldots, \mathcal{R}_{m}+\mathbf{c}_{m}\right\}$ be the family of $i$-components defined above, $m \geq 4$, and let $\sigma_{1}, \sigma_{2}, \ldots, \sigma_{m}$ be $m$ permutations of the elements in $\mathbb{F}_{q}, \sigma_{i}(1) \neq 1$ for all $1 \leq i \leq m$. Then the code

$$
\mathcal{C}=\left(\mathcal{H}_{q, m} \backslash \bigcup_{i=1}^{m} \mathcal{R}_{i}+\mathbf{c}_{i}\right) \bigcup\left(\bigcup_{i=1}^{m} \sigma_{i}\left(\mathcal{R}_{i}+\mathbf{c}_{i}\right)\right)
$$

is a full-rank 1-perfect code over the finite field of characteristic 2 .

Proof: Lemma 3 implies that the family $\mathcal{F}$ is an admissible family of $i$-components of the Hamming code $\mathcal{H}_{q, m}$. Therefore by Theorem 6 we obtain that the $\operatorname{code} \mathcal{C}$ is a $q$-ary 1perfect code of length $n=q^{m}-1 / q-1$. From the definition of a Hamming code $\mathcal{H}_{q, m}$ it follows that $\operatorname{rank}\left(\mathcal{H}_{q, m}\right)=n-m$. The dimension of $\mathcal{R}_{i}$ is $q^{m-1}-1$. Taking into account that

$$
\begin{aligned}
\mid \mathcal{H}_{q, m} & \backslash \bigcup_{i=1}^{m} \mathcal{R}_{i}+\mathbf{c}_{i} \mid \\
& =q^{n-m}-m q^{q^{m-1}-1}>\frac{1}{q} \cdot q^{n-m}=\frac{1}{q} \cdot\left|\mathcal{H}_{q, m}\right| .
\end{aligned}
$$

We have

$$
\operatorname{rank}\left(\mathcal{H}_{q, m} \backslash \bigcup_{i=1}^{m} \mathcal{R}_{i}+\mathbf{c}_{i}\right)=n-m .
$$

By construction of vectors $\mathbf{c}_{1}, \mathbf{c}_{2}, \ldots, \mathbf{c}_{m}$, we have that the vectors

$$
\mathbf{c}_{1}\left(1, \sigma_{1}\right), \mathbf{c}_{2}\left(2, \sigma_{2}\right), \ldots, \mathbf{c}_{m}\left(m, \sigma_{m}\right)
$$

are linearly independent. We remind that, by definition,

$$
\mathbf{c}(i, \sigma)=\left(c_{1}, c_{2}, \ldots, \sigma\left(c_{i}\right), \ldots, c_{n}\right) .
$$

Since $\sigma_{i}(1) \neq 1$ for all $1 \leq i \leq m$, we have

$$
\left\{\mathbf{c}_{1}\left(1, \sigma_{1}\right), \mathbf{c}_{2}\left(2, \sigma_{2}\right), \ldots, \mathbf{c}_{m}\left(m, \sigma_{m}\right)\right\} \cap \mathcal{H}_{q, m}=\varnothing .
$$

But

$$
\left\{\mathbf{c}_{1}\left(1, \sigma_{1}\right), \mathbf{c}_{2}\left(2, \sigma_{2}\right), \ldots, \mathbf{c}_{m}\left(m, \sigma_{m}\right)\right\} \subset \mathcal{C} .
$$

Therefore, $\operatorname{rank}(\mathcal{C})=n$.

Finally, we present a modification of the Etzion and Vardy construction of the full-rank 1-perfect codes for finite fields of arbitrary characteristic. We define

$$
\begin{aligned}
\mathbf{c}_{1}= & \xi\left(\mathbf{h}_{1}\right)+\xi\left(\mathbf{h}_{1}+\mathbf{h}_{2}+\mathbf{h}_{3}\right)-\xi\left(\mathbf{h}_{1}+\mathbf{h}_{2}-\mathbf{h}_{4}\right)- \\
& \xi\left(\mathbf{h}_{1}+\mathbf{h}_{3}+\mathbf{h}_{4}\right) \\
\mathbf{c}_{2}= & \xi\left(\mathbf{h}_{1}\right)+\xi\left(\mathbf{h}_{2}\right)-\xi\left(\mathbf{h}_{1}-\mathbf{h}_{3}-\mathbf{h}_{4}\right)- \\
& \xi\left(\mathbf{h}_{2}+\mathbf{h}_{3}+\mathbf{h}_{4}\right) \\
\mathbf{c}_{4}= & \xi\left(\mathbf{h}_{1}\right)-\xi\left(\mathbf{h}_{2}\right)-\xi\left(\mathbf{h}_{3}\right)+\xi\left(\mathbf{h}_{4}\right)+ \\
& \xi\left(\mathbf{h}_{1}+\mathbf{h}_{2}+\mathbf{h}_{3}\right)-\xi\left(\mathbf{h}_{1}+\mathbf{h}_{2}+\mathbf{h}_{4}\right)- \\
& \xi\left(\mathbf{h}_{1}+\mathbf{h}_{3}+\mathbf{h}_{4}\right)+\xi\left(\mathbf{h}_{2}+\mathbf{h}_{3}+\mathbf{h}_{4}\right) .
\end{aligned}
$$

Further let $j \in\{1,2, \ldots, m\} \backslash\{1,2,4\}$. If $j$ is odd, define

$$
\mathbf{c}_{j}=\sum_{i=1}^{j} \xi\left(\mathbf{h}_{i}\right)-\xi\left(\mathbf{h}_{1}+\mathbf{h}_{2}+\cdots+\mathbf{h}_{j}\right) .
$$

Otherwise set

$$
\begin{array}{r}
\mathbf{c}_{j}=\sum_{i=1}^{j} \xi\left(\mathbf{h}_{i}\right)-\xi\left(\mathbf{h}_{1}+\mathbf{h}_{2}+\cdots+\mathbf{h}_{j / 2}\right)- \\
\xi\left(\mathbf{h}_{j / 2+1}+\mathbf{h}_{j / 2+2}+\cdots+\mathbf{h}_{j}\right) .
\end{array}
$$

Since the columns $\mathbf{h}_{\mathbf{1}}, \mathbf{h}_{\mathbf{2}}, \ldots, \mathbf{h}_{\mathbf{m}}$ are linearly independent it follows that $\mathbf{c}_{i} \in \mathcal{H}_{q, m}, i=1,2, \ldots, m$. Further we define a family $\mathcal{F}=\left\{\mathcal{R}_{1}+\mathbf{c}_{1}, \mathcal{R}_{2}+\mathbf{c}_{2}, \ldots, \mathcal{R}_{m}+\mathbf{c}_{m}\right\}$ of $i$-components of the Hamming code $\mathcal{H}_{q, m}$.

Theorem 8: Let $\mathbb{F}_{q}$ be a finite field, let $\mathcal{F}=\left\{\mathcal{R}_{1}+\mathbf{c}_{1}, \mathcal{R}_{2}+\right.$ $\left.\mathbf{c}_{2}, \ldots, \mathcal{R}_{m}+\mathbf{c}_{m}\right\}$ be the family of $i$-components defined above, $m \geq 4$, and let $\sigma_{1}, \sigma_{2}, \ldots, \sigma_{m}$ be $m$ permutations of the elements in $\mathbb{F}_{q}, \sigma_{i}(1) \neq 1$ for all $1 \leq i \leq m$. Then the code

$$
\mathcal{C}=\left(\mathcal{H}_{q, m} \backslash \bigcup_{i=1}^{m} \mathcal{R}_{i}+\mathbf{c}_{i}\right) \bigcup\left(\bigcup_{i=1}^{m} \sigma_{i}\left(\mathcal{R}_{i}+\mathbf{c}_{i}\right)\right)
$$

is a full-rank 1-perfect code over the finite field.

This theorem is proved exactly like Theorem 7 .

\section{CONCLUSION}

Etzion and Vardy [1] discovered a peculiar method to construct an admissible family of $i$-components of the binary Hamming code. In [20], the method of Etzion and Vardy has been generalized to $q$-ary codes. Besides the method of Etzion and Vardy, there is also method to construct an admissible family of $i$-components of the Hamming code suggested in [5], [15]-[18]. In [15], sufficient conditions for disjointness of $i$-components of a binary Hamming code are obtained. By means of these conditions, the nonsystematic binary 1perfect codes of length $\mathrm{n}=15$ were constructed, see [16]. Also in [17], the binary 1-perfect codes with trivial kernel were constructed by means of the sufficient conditions from [15]. In [5], a criterion for disjointness of the $i$-components of a binary Hamming code is obtained, and regular partitions of the binary Hamming codes into $i$-components with new 
parameters are constructed. In [18], the results from [5] have been generalized to $q$-ary codes.

Etzion [2] generalized some results of [1] to $q$-ary case. Etzion [2] defined the $i$-components of a $q$-ary Hamming code in terms of the generator matrices and constructed $q$ ary 1 -perfect codes by switching $i$-components of the $q$-ary Hamming code for fixed coordinate $i$.

\section{REFERENCES}

[1] T. Etzion and A. Vardy, "Perfect binary codes: Constructions, properties, and enumeration," IEEE Trans. Inform. Theory, vol. 40, no. 3, pp. 754763, May 1994.

[2] T. Etzion, "Nonequivalent $q$-ary perfect codes", SIAM Journal of Discrete Mathematics, vol. 9, no. 3, pp. 413-423, August 1996.

[3] O. Heden, "A new construction of group and nongroup perfect codes," Inform. and Control, vol. 34, pp. 314-323, 1977.

[4] O. Heden and D. S. Krotov, "On the structure of non-full-rank perfect codes," Adv. Math. Commun., vol. 5, no. 2, pp. 149-156, May 2011.

[5] S. A. Malyugin and A. M. Romanov, "On partitions of the Hamming code into disjoint components," (in Russian), Diskretn. Anal. Issled. Oper., Ser. 1, vol. 9, no. 1, pp. 42-48, 2002.

[6] B. Lindström, "On group and nongroup perfect codes in $q$ symbols," Math. Scand., vol. 25, pp. 149-158, 1969.

[7] J. H. van Lint, "Nonexistence theorems for perfect error-correctingcodes," Computers in Algebra and Number Theory, vol. IV, SIAM-AMS Proceedings, 1971.

[8] P. R. J. Östergård and O. Pottonen, "The perfect binary one-errorcorrecting codes of length 15: Part I-Classification," IEEE Trans. Inform. Theory, vol. 55, no 10, pp. 4657-4660, Oct. 2009.

[9] P. R. J. Östergård, O. Pottonen, and K. T. Phelps, "The perfect binary one-error-correcting codes of length 15: Part II-Properties," IEEE Trans. Inform. Theory, vol. 56, no. 6, pp. 2571-2582, June 2010.

[10] K. T. Phelps, "A combinatorial construction of perfect codes," SIAM J. Algebraic Discrete Methods, vol. 4, pp. 398-403, 1983.

[11] K. T. Phelps and M. LeVan, "Switching equivalence classes of perfect codes," Designs, Codes Cryptogr., vol. 16, pp. 179-184, 1999.

[12] K. T. Phelps and M. LeVan, "Kernels of nonlinear Hamming codes," Designs, Codes Cryptogr., vol. 6, no. 3, pp. 247-257, 1995.

[13] K. T. Phelps and M. Villanueva, "Ranks of $q$-ary 1-perfect codes," Designs, Codes and Cryptogr., vol. 27, no. 12, pp. 139-144, 2002.

[14] K. T. Phelps, J. Rifà, and M. Villanueva, "Kernels and $p$-kernels of $p^{r}$ ary 1-perfect codes," Designs, Codes and Cryptogr., vol. 37, no. 2, pp. 243-261, 2005.

[15] A. M. Romanov, "On construction of nonlinear perfect binary codes by inversion of symbols," (in Russian), Diskretn. Anal. Issled. Oper., Ser. 1, vol. 4 no. 1, pp. 46-52, 1997.

[16] A. M. Romanov, "On nonsystematic perfect binary codes of length 15," (in Russian), Discretn. Anal. Issled. Oper. Ser. 1, vol. 4, no. 4, pp. 7578, 1997. English translation in Discrete Appl. Math., vol. 135, no. 1-3, pp. 255-258, 2004.

[17] A. M. Romanov, "Perfect binary codes with trivial kernel," (in Russian), Diskretn. Anal. Issled. Oper., Ser. 1, vol. 7, no. 2, pp. 71-74, 2000.

[18] A. M. Romanov, "On partitions of $q$-ary Hamming codes into disjoint components," (in Russian), Diskretn. Anal. Issled. Oper., Ser. 1, vol. 11, no. 3, pp. 80-87, 2004.

[19] A. M. Romanov, "A survey of methods for constructing nonlinear perfect binary codes," (in Russian), Diskretn. Anal. Issled. Oper., Ser. 1, vol. 13, no 4, pp. 60-88, 2006. English translation in J. Appl. Industr. Math., vol. 2, no. 2, pp. 252-269, 2008.

[20] A. M. Romanov, "On admissible families of components of Hamming codes," (in Russian), Diskretn. Anal. Issled. Oper., vol. 19, no. 2, pp. 84-91, 2012. English translation in J. Appl. Industr. Math., vol. 6, no. 3, pp. 355-359, 2012.

[21] A. M. Romanov, "Hamiltonicity of minimum distance graphs of 1perfect codes," Electron. J. Comb., vol. 19, no. 1, P65, 2012.

[22] F. I. Solov'eva, "On binary nongroup codes," (in Russian), Methodi Diskr. Analiza, vol. 37, pp. 65-76, 1981.

[23] J. Schönheim, "On linear and nonlinear single-error-correcting $q$-nary perfect codes," Inform. and Control, vol. 12, pp. 23-26, 1968.

[24] A. Tietäväinen, "On the nonexistence of perfect codes over finite fields," SIAM J. Appl. Math., vol. 24, no. 1, pp. 88-96, Jan. 1973.

[25] J. L. Vasil'ev, "On nongroup close-packed codes," (in Russian), Probl. Kibemet., vol. 8, pp. 375-378, 1962.
[26] J. L. Vasil'ev, personal communication.

[27] V. A. Zinov'ev and V. K. Leont'ev, "The nonexistence of perfect codes over Galois fields," (in Russian), Probl. Control and Info. Theory, vol. 2, pp. 123-132, 1973. 